\title{
Detecting Physical Abilities through Smartphone Sensors: An Assistive Technology Application
}

\author{
Paul Whittington, Huseyin Dogan, Keith Phalp, Nan Jiang
}

Faculty of Science \& Technology, Bournemouth University, Poole, UK

Dr Paul Whittington, P319, Poole House, Talbot Campus, Bournemouth University, Fern Barrow, Poole Dorset, BH12 5BB

Email: whittingtonp@bournemouth.ac.uk.

Tel: $+44(0) 1202967224$

Dr Paul Whittington is a Postdoctoral Researcher in Assistive Technologies in the Faculty of Science \& Technology at Bournemouth University. Dr Whittington's research focuses on Assistive Technologies, Human Factors, Usability Engineering and System of Systems. He is a member of the Human Computer Interaction (HCI) Research Group and has previously been involved with the MSc Human Factors and Usability Engineering courses. Dr Whittington received his Doctorate (PhD) in Human Computer Interaction from Bournemouth University in 2017 and BSc (Hons) in Computing also from Bournemouth University in 2013. During his doctorate (funded by a Vice-Chancellor's scholarship), he developed the SmartAbility Framework which recommends technologies based on the actions (user abilities) that people with reduced physical abilities (disabilities) can perform independently. Dr Whittington's postdoctoral research is developing a SmartAbility Application to compliment the Framework. The Application will automatically detect user abilities and recommend suitable Assistive Technologies. The aim of the Application to increase the awareness of Assistive Technology, that can provide potential increases in quality of life, for people with reduced physical abilities. It is anticipated that the Application will evolve into a product promotion tool for charities and industries associated with Assistive Technologies.

Dr Huseyin Dogan is a Principal Academic in Computing at Bournemouth University (BU). Dr Dogan's research focuses on Human Factors, Assistive Technology, Digital Health and Systems Engineering. He is the Co-Founder and Co-Chair of the Human Computer Interaction (HCI) research group and previously led the postgraduate courses (2012-2015) and then the undergraduate courses (2015-2017) in Computing at BU, managing over 900 students. Dr Dogan received his Engineering Doctorate (EngD) in Systems Engineering from Loughborough University, MSc in Human Computer Interaction with Ergonomics from University College London, and BSc in Computer Science from Queen Mary University of London. Dr Dogan worked on a number of research projects funded by the industry, Innovate UK, Engineering and Physical Sciences Research Council (EPSRC), European Commission (EC) and Higher Education Innovation Fund (HEIF). Some examples include FP7 OASIS (Open Advanced System for crisIS management); FP7 COPE (Common Operational Picture Exploitation); FP7 T-AREA-SoS (Trans-Atlantic Research and Education Agenda in System of Systems); and HEIF-6 cFACETS (Fatigue: Applying Cognitive behavioural and Energy effectiveness Techniques to lifeStyle). Prior to BU, he worked as a Research Associate at Loughborough University. He has 8 years industrial experience working for BAE Systems Advanced Technology Centre. 


\section{Detecting Abilities through Smartphone Sensors}

Dr Nan Jiang joined Bournemouth University in 2010 and he is an Associate Professor in Human Computer Interaction. Prior to this, Nan was an Open Distance Learning (ODL) tutor at Queen Mary, University of London, where he also received his PhD in Web Usability and MSc in Advanced Methods. Nan has 10 years of full stack web development experience as a freelance technical consultant and technical writer. He is specialised in web/mobile architectural and interface design but he also provides technical support on backend development. He has provided consultancy for many companies and organisations through a number of industrial and research projects funded by European Regional Development Fund (ERDF), European Commission Horizon 2020 (H2020), Higher Education Innovation Funding (HEIF) and Bill \& Melinda Gates Foundation. Nan was also a public invited expert for W3C HTML Working Group and he is still a member of W3C China. Nan mainly teaches user centred design and web development. He currently has two main roles: Deputy Head of Department and Programme Leader for MSc Information Technology. In the past, he worked as the Final Year Project Coordinator managing over 800 final year students between 2013 and 2018 and led MSc Enterprise Information System.

Professor Keith Phalp is Executive Dean of the Faculty of Science and Technology at Bournemouth University, and Professor of Software Engineering. Previous leadership roles have included Head of Software Systems and Psychology, Head of Computing and Informatics, and Deputy Dean for Education and Professional Practice. His research foci cover software engineering, particularly the early phases of software projects and the relationship between business and software models, model driven development, applications of AI, and, in recent years, social computing, which encompasses digital addiction and on-line gambling. He has extensive project leadership expertise, having led major successful EC funded projects, as well as Knowledge Transfer Projects, and his research has brought tangible and financial benefits to the organisations involved. 


\section{Detecting Physical Abilities through Smartphone Sensors: An Assistive Technology Application}

Purpose: It is important to promote assistive technologies to improve quality of life. The proposed SmartAbility Android Application recommends assistive technologies for people with reduced physical abilities, by focusing on actions that can be performed independently.

Materials and methods: The SmartAbility Application uses Android built-in sensors, e.g. accelerometer and gyroscope and application programming interfaces (APIs) to detect physical abilities, e.g. head movements and blowing and recommend suitable assistive technologies. This is supported by a MySQL database that stores assistive technologies and mappings between abilities. The underpinning research is the SmartAbility Framework that culminates the knowledge obtained during previously feasibility trials and usability evaluations.

Results: The Application was evaluated by pupils $(\mathrm{n}=18)$ at special educational needs schools with physical conditions, including cerebral palsy, autism and Noonan syndrome, and assessed through the NASA Task Load Index (TLX) and System Usability Scale. Analysis using the Adjective Rating Scale highlighted that the Application achieves 'Good Usability'.

Conclusion: The SmartAbility Application demonstrates that built-in sensors of Android devices and their APIs, can detect actions that users perform, e.g. head movements and speaking. The Application contains a database where assistive technologies are mapped to physical abilities, in order to provide suitable recommendations. It will be disseminated to assistive technology charities and manufacturers and be used by healthcare professionals as part of the rehabilitation process. Future developments of SmartAbility include the creation of a second Application designed specifically to recommend assistive technologies for the education sector, based on users' physical and cognitive abilities. 
Detecting Abilities through Smartphone Sensors

Keywords: assistive technology; disability; human computer interaction; human factors; interactive systems; usability engineering; user interfaces

\section{Introduction}

According to The World Bank (2020), there are one billion people (15\%) worldwide, who have disabilities affecting their daily interactions with society. Therefore, it is important to promote assistive technologies to improve quality of life, which is an ever increasing market (Gallagher and Petrie 2013)0. It was evident from interactions with the user community during our previous research, that people with physical disabilities, which we refer to as, 'reduced physical abilities' can be unaware of the assistive technologies that are available to provide assistance and an improved quality of life.

We believe that this awareness can be achieved by adopting an ability based design approach (Wobbrock et al. 2011) that focuses on abilities of the user in the design process of interactive systems, to create a system that reflects the full range of human potential. This therefore focuses on ability rather than disability. Ability based design motivated the development of the SmartAbility Framework to provide recommendations of assistive technologies based on users' physical abilities, i.e. actions that users can perform independently. The framework concept was established in 2013 and evolved during four years of PhD research (Whittington and Dogan 2016) and enhanced through three years of ongoing Postdoctoral Research (Whittington et al. 2018). During this period, four controlled usability evaluations were conducted as well as two framework validations. This culminated in the involvement of approximately 100 participants, including those with reduced physical and cognitive abilities and domain experts with assistive technology, human computer interaction and healthcare backgrounds. 
The SmartAbility Framework consists of six elements that are mapped together to form the relationship between Physical Conditions and Assistive Technologies. It is evident from discussions with the user community that there is a need for an assistive technology recommendation system to be developed. The Framework evolved from previously conducted requirements elicitations, feasibility trials, controlled usability evaluations and validation (Whittington and Dogan 2015). The initial requirements elicitation phase identified the tasks that were challenging for the user community (Whittington et al. 2018), followed by the feasibility trials of technologies to determine their potential in providing assistance with daily tasks. The Framework was subsequently developed by analysing physical conditions through the International Classification of Functioning (ICF), Disability and Health Framework (World Health Organization 2001). This resulted in the identification of physical conditions that could adversely affect user abilities. The rationale behind investigating users' physical abilities originated from the conduction of a head tracking experimentation involving iOS Switch Control (Whittington and Dogan 2016), where it could only be operated by users who possess full neck Range of Motion (ROM). This emphasises that ROM is one of the determining factors for the suitability of assistive technology, along with other physical abilities, such as eye movements. The Framework was validated through the involvement of the intended user community and domain experts with technology or healthcare backgrounds. The validation revealed a number of modifications that needed to be made to the Framework, including a reduction in the number of elements, enhanced mappings through the adoption of symbols derived from Quality Function Deployment (QFD) (Akao 1990) and simplification of the terminology.

This paper focuses on the development and subsequent evaluation of the SmartAbility Application, where users' physical abilities are detected through sensors that 
are built into Android devices. Android was selected as the platform for the Application as it has $73 \%$ of the worldwide market share (StatCounter 2020). The use of built-in sensors minimises the level of manual input required for people with reduced physical abilities to discover assistive technologies. The Application can be used by charity representatives or healthcare professionals (HCPs) during the rehabilitation of clients with reduced physical abilities, to provide recommendations of suitable assistive technologies.

An overview of the Framework is provided, as well as a systematic literature review of built-in sensor technologies in smart devices, based on four research questions we previously defined (Whittington et al. 2018). The methodology and NASA Task Load Index and System Usability Scale results of a usability evaluation are also presented.

\section{Related Work}

During the development of the SmartAbility Framework, the initial literature review (Whittington and Dogan 2016a, Whittington and Dogan 2016b) was conducted into domains of Physical Conditions, Abilities, Interaction Modalities, Human-Centred Design (HCD). To devise the first two elements of the Framework (Physical Conditions and Abilities), existing classifications of disability and measurements of user abilities were analysed. The Interaction Mediums and Technologies elements were established through reviewing currently available assistive technologies and conducting feasibility trials and controlled usability evaluations (Whittington et al. 2018). It was essential to follow an HCD approach, as the intended user community for the SmartAbility Framework, i.e. people with reduced physical abilities and domain experts from healthcare and technology, were involved during the development. Prior to the development of the SmartAbility Android Application, a second systematic literature review was undertaken into built-in sensors of 
Detecting Abilities through Smartphone Sensors

smart devices (Whittington et al. 2018).

\section{Physical Conditions}

To establish a common language for defining disability, the World Health Organization (2001) developed the ICF as a worldwide standard for disability classification. Their aim was to ensure that disability be viewed as "a complex interaction between the person and their environment" instead of characterising individuals (Kostanjsek 2011). R. Andrews (personal communication, 2014) compared the relationship between the ICF, Downton Scale and the impairment types of 'Motor Control', 'Senses' and 'Cognitive Ability'. The key finding was that there were a variety of impairments as a result of disabilities, such as speech impediment and reduced movement. This literature formed the basis of the Physical Conditions element of the SmartAbility Framework, where conditions (such as cerebral palsy) were mapped to their associated impairments. ROM (Keilhofner 2006) was considered as an important factor to identify the movements that users can perform independently. This was supported by the results of an experiment involving users with reduced physical ability, interacting with head tracking technology (Whittington and Dogan 2016b). It was established that users required a full $80^{\circ}$ range of neck movement for successful interaction. Usually, ROM is measured using a goniometer to provide an assessment of the extent to which the users can move their joints between $0^{\circ}$ and $360^{\circ}$. However, for the purpose of the SmartAbility Framework, it is sufficient to categorise ROM and other user abilities into three Likert Ease of Action scales; 'Easy', 'Difficult' and 'Impossible' based on literature discussed in the following section. 
Detecting Abilities through Smartphone Sensors

\section{Abilities}

The ability-based design concept (Wobbrock et al. 2018) was adopted to develop the SmartAbility Framework, as the potential of humans is maximised through focusing on their abilities. Ability-based design was based on the seven principles originally defined by Kelley and Hartfield (2006) as (1) Ability, (2) Accountability, (3) Adaptation, (4) Transparency, (5) Performance, (6) Context and (7) Commodity. Principle (3) can also be applied, whereby the assistive technologies recommended by the Framework should be adaptable to suit the users' abilities. It is important to take into consideration the Commodity principle when recommending technologies, as the recommendations should be affordable for the intended user community. This is emphasised by Sloan et al. (2010), who state these factors are usually 'barriers to progress for assistive technologies'. There has been previous research conducted into the ROM required for performing daily living activities, which was applied to determine the 'Easy', 'Difficult' and 'Impossible' categories of Ease of Action for the SmartAbility Framework. Relating to the upwards and downwards tilting of the head, Gates et al. (2015) quantified that the acceptable ROM was between $0^{\circ}$ and $108^{\circ}$. Gates et al. (2015) also conducted a similar study into turning the head left and right, where the acceptable ROM was determined to be between $-65^{\circ}$ and $105^{\circ}$. For tilting the head, the boundary for the 'Easy' category was set to above $60^{\circ} \mathrm{ROM}$, the 'Difficult' category was set below $60^{\circ} \mathrm{ROM}$ and the 'Impossible' category set to below $10^{\circ}$ ROM. For turning the head, the boundary for the 'Easy' category was set to above $50^{\circ}$ and below $-40^{\circ} \mathrm{ROM}$, the 'Difficult' category was set between $10^{\circ}$ and $50^{\circ} \mathrm{ROM}$ and between $-40^{\circ}$ and $-10^{\circ} \mathrm{ROM}$ and the 'Impossible' category was set to between $-10^{\circ}$ and $10^{\circ}$ ROM to cater for errors. 
Khadilkar et al. (2014) identified that the minimum shoulder flexion required for daily living activities was $118^{\circ} \mathrm{ROM}$ and this value determined the lower boundary for the 'Easy' category for shoulder movements. The boundaries for the 'Difficult' category were set between $10^{\circ}$ and $118^{\circ} \mathrm{ROM}$ and the boundary for the 'Impossible' category was set to below $10^{\circ}$ ROM to cater for errors. The study by Gates et al. (2015) also stated that the ROM for the elbow was between $0^{\circ}$ and $121^{\circ}$ for movement required in daily living activities, which denotes the 'Easy' category. Therefore, the 'Difficult' category was established between $10^{\circ}$ and $121^{\circ}$ ROM and the 'Impossible' boundary was set to less than $10^{\circ} \mathrm{ROM}$, to allow for capture of erroneous data.

The required ROM to perform living activities involving the wrist is between $-40^{\circ}$ of flexion and $38^{\circ}$ of extension (Gates et al. 2015), where flexion refers to the bending of the wrist in a downwards direction and extension in an upwards direction. The 'Easy' category was defined between this range, 'Difficult' when the user is only able to achieve $-40^{\circ}$ of flexion and $38^{\circ}$ of extension and 'Impossible' when the user can only move their shoulder by less than $10^{\circ}$ flexion or extension.

The ROM required to perform activities with the ankle is defined by Brockett and Chapman (2016), where it is stated that the ROM required for walking, ascending and descending the stairs is $30^{\circ}, 37^{\circ}$ and $56^{\circ} \mathrm{ROM}$ respectively. Therefore, the ROM boundary for ankle movements in the SmartAbility Application was set to $56^{\circ}$ indicating the 'Easy' category. The 'Difficult' category implies that the user is not able to achieve this upper boundary and Impossible when less than $10^{\circ}$ ROM is detected.

Touch gestures are used to determine the abilities of fingers and thumbs. The Application can detect the gestures of drag, press, swipe, single and multi-tap, as these are 
commonly used when interacting with smart devices. These abilities are measured in terms of whether the user can or cannot perform each gesture.

\section{Interaction Mediums and Technologies}

In the Framework we define Interaction Mediums as specific parts of the body used to control assistive technologies, where assistive technologies are recommended to, "increase, maintain, or improve the functional capabilities of persons with disabilities" (Assistive Technology Industry Association 2020). According to Biswas and Robinson (2008), people with reduced physical abilities usually have a more diverse ROM compared to able-bodied users. Therefore, it is typical that these users can require more advanced interaction mediums for assistive technologies instead of traditional touch-based interaction. Sip-andPuff is an assistive technology that sends signals to a device using air pressure by sucking in (sipping) or blowing out (puffing) on a straw or tube. This is used by people who have insufficient finger dexterity to operate a joystick (Origin Instruments Corporation 2020). Electroencephalogram (EEG) is another alternative interaction medium that provides braincomputer interaction through the attachment of electrodes to the scalp. Head-mounted displays could also be utilised as assistive technologies. The Interaction Mediums and Technologies elements include products that conform to the Commodity principle of ability-based design.

\section{Smart Device Sensors}

Smart devices contain built-in sensors that detect their location and motion, as well as the physical characteristics of the user. The sensors include an accelerometer and gyroscope, which combine to provide an accurate measurement of orientation and motion of the 
device.

Face detection is another capability of smart devices where users' faces can be distinguished from real-time video and images, including their orientation, facial expressions and landmarks, such as the eyes and mouth. On the Android platform, this functionality is achieved through the Face Detection Application Programming Interface, known as an API (Google Developers 2020). When a face is detected, a 'Face' object is instantiated by executing methods to obtain the position, width and height. The getEulerY() and getEulerZ() enable the orientation and rotation of the face to be determined. There are specific methods to detect face expressions, including getIsSmilingProbability() and getIsLeftEyeOpenProbability().

The touchscreen can also be viewed as a sensor that detects touch gestures involving one or more fingers. The step sensor detects a number of steps taken by the user in a specific timeframe, whereas the accelerometer and gyroscope can capture the user's ability to move their arms.

\section{Current Physical Ability Assessment Methods}

During the validation of the SmartAbility Framework, it became apparent that people with reduced physical abilities were often unaware of the assistive technologies that are available to provide support in their lives. Assistive technology charities highlighted that there is not a common repository of assistive technologies available, resulting in a lack of awareness.

Currently, charities recommend assistive technologies to clients after a manual assessment of their abilities. Discussions with charities identified that the first stage of this process is to perform a telephone conversation to discuss a client's requirements for 
assistive technology. Referral forms would then be sent to HCPs, such as Occupational Therapists and Physiotherapists. Assessment visits to clients would take place to gain a detailed understanding of their requirements for assistive technology. The Occupational Therapist or Physiotherapist and a representative from the charity would be present at the visit. The objective of the visit is to recommend suitable assistive technologies to improve the client's quality of life.

The charities find the manual assessment process time consuming and costly, as a result of travelling to clients. These assessments also have the limitation of being subjective and dependent on the knowledge of the HCPs and charity representatives, in terms of the available assistive technologies.

The SmartAbility Application provides an alternative, automated method of assessing user abilities, through a smartphone. It is anticipated that this would be less time consuming than the current manual assessment methods.

\section{SmartAbility Framework}

The Android Application is based on the SmartAbility Framework that provides assistive technology recommendations based on the physical abilities of the user, i.e. actions that can be performed independently without assistance. Previously conducted requirements elicitation (Whittington et al. 2015b), technology feasibility trials and controlled usability evaluations (Whittington et al. 2015a) contributed to the underpinning knowledge of the Framework. Results of these evaluations have previously been published in IEEE Transactions in Human-Machine Systems (Whittington and Dogan 2016). The development of the Framework consisted of three iterations, each with a validation phase involving the user community of people with reduced physical abilities and domain experts. 
Detecting Abilities through Smartphone Sensors

The mappings between the Abilities, Interaction Mediums and Technologies elements in Version 2, consist of colour-coded symbols, checkmarks and Likert scales (Whittington et al. 2018). To supplement the Framework, a holistic model (shown in Figure 1) was developed based on the House of Quality (HoQ) matrix of the Quality Function Deployment (QFD) tool (Akao 1990).

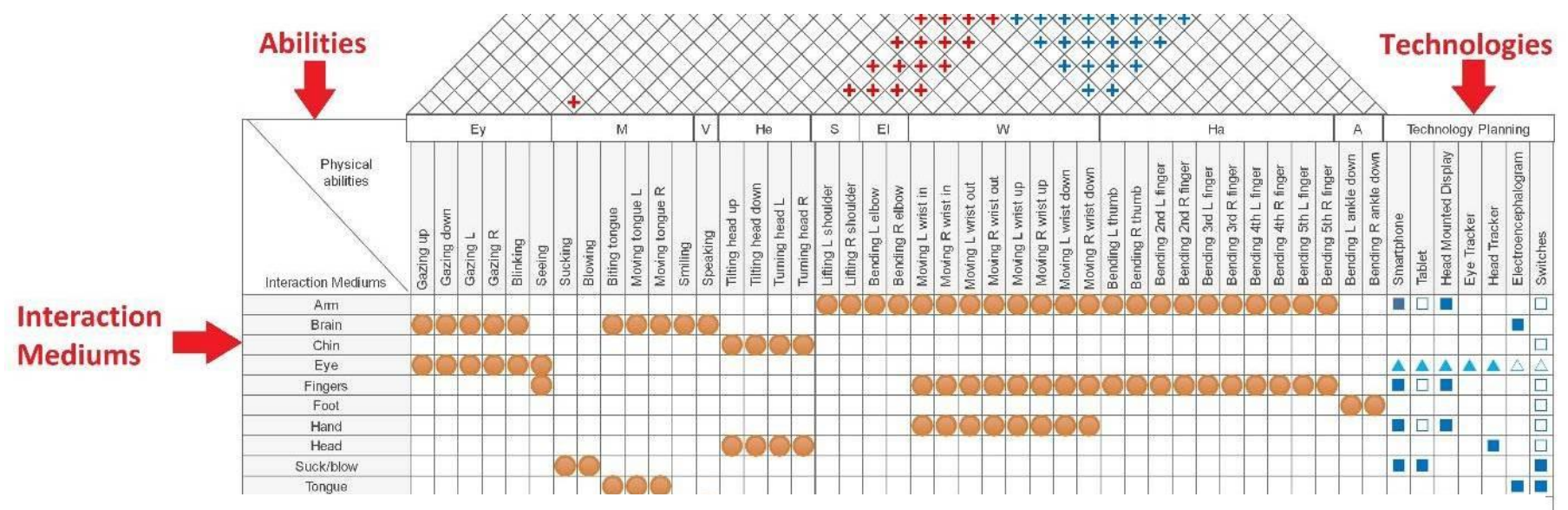

Figure 1. An Extract of the SmartAbility Holistic Model based on the Quality Function Deployment Concept

\section{Framework Elements}

The SmartAbility Framework contains four elements: Physical Conditions, Abilities, Interaction Mediums and Technologies. Each element is mapped onto the subsequent element and contains images to assist with user understanding.

\section{Physical Conditions}

Element 1 considers the range of physical conditions that result in reduced physical abilities to filter the conditions into generic categories. Based on observations from previously conducted evaluations and literature, the Physical Conditions are mapped onto 
Detecting Abilities through Smartphone Sensors

Abilities. The physical condition of the user is the input to this element, which is then used to establish the abilities that may be affected, therefore informing the Abilities element.

\section{Abilities}

This element (shown in Figure 2) considers how a user's physical ability to perform certain movements is affected by their physical condition. There are three categories: 'Easy', 'Difficult' and 'Impossible', which are illustrated by a traffic light style grading system of green, amber and red. To establish the definition of the three categories, literature referring to ROM required for daily activities was analysed, as described in Section II (B). The 'Easy' category is when the user can meet the defined ranges, 'Difficult' when the user is only able to achieve the boundaries and 'Impossible' when the user cannot perform the ability. Users select the categories which best describe their abilities, to form the inputs to the Interaction Mediums element, to enable recommendations to be made.

\section{Interaction Mediums}

Element 3 concerns the abilities that are required through different interaction mediums. The QFD approach was adopted to create two symbols that indicate mandatory or nonmandatory abilities for successful interaction, by a solid orange symbol and a white-centred symbol respectively. It is imperative that the user is able to perform all of the mandatory abilities, whereas the user must only possess at least one of the non-mandatory abilities. The interaction mediums suitable for a user are the output of the element, leading to the final Technologies element. 


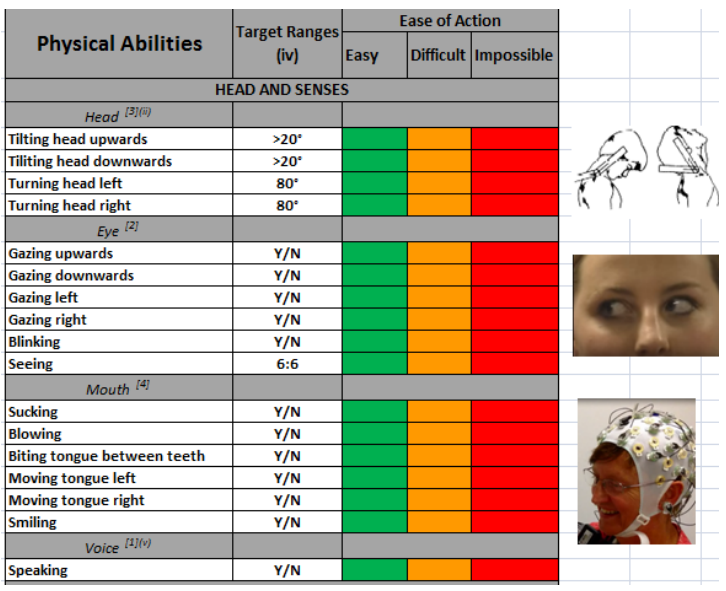

Figure 2. The Abilities Element of the SmartAbility Framework

\section{Technologies}

The Interaction Mediums are mapped to assistive technologies, based on literature reviews and results from previously conducted feasibility trials and usability evaluations. There are six colour-coded symbols indicating the levels of physical agility (motor skills), visual acuity (sight) and speech clarity required for successful operations.

The recommendations of Interaction Mediums and Technologies are achieved through mappings between the elements. As an example, a Sip-and-Puff interaction medium relies on the user to have the abilities to both suck in and blow out. The Framework would therefore only recommend 'Sip 'n Puff' for users who have these two abilities.

An example of use for the Framework would be for an individual with cerebral palsy who is not able to bend their fingers or speak. In the Abilities element, they would indicate that these abilities are 'Impossible' for them to perform. The Framework would therefore recommend brain, chin, eye, foot, head, Sip-and-Puff and tongue-based interaction mediums, which can be used to control a smartphone, tablet, head mounted display, eye tracker, head tracker, electroencephalogram or switch. 
Detecting Abilities through Smartphone Sensors

\section{SmartAbility Application Development}

The key aspects to consider for the development of the SmartAbility Application were the use of smart device sensors to detect user abilities, user interface design and the assistive technology database structure.

\section{Methodology}

Prior to the development of the SmartAbility Application, a systematic literature review was conducted into smart device sensors. The review contained Scoping and Planning stages, as described by Siddaway (2020). The Scoping stage defined the aim of the literature review by establishing four research questions:

(1) How can user abilities be detected through Android and iOS sensors?

(2) Which mobile operating systems support the detection of user abilities?

(3) Are there user abilities that cannot currently be detected through Android and iOS devices?

(4) How can ability-based smart device sensors be identified and mapped to the SmartAbility Framework?

An analysis was conducted into previous systematic literature reviews in the domain. It was established that reviews have been conducted into the application of smart device sensors in healthcare to monitor patients and for fall detection to determine sudden changes in velocity using accelerometers. However, there had not been a systematic literature review conducted to map assistive technologies to user abilities.

In the Planning stage, search terms, inclusion and exclusion criteria were established. Example search terms used for question 1 were 'smartphone accelerometer' and 'user 
Detecting Abilities through Smartphone Sensors

abilities sensors' and for question 2, 'android smiling detection' and 'android detect suck blow'. There were 15 inclusion criteria defined to ensure that the literature review obtained materials that were relevant to the research questions. Two of the inclusion criteria were: 'The sensor technologies can be implemented on a smartphone platform' and 'The sensors are monitoring one or more human movements'. Ten exclusion criteria were established and included, 'The sensors monitor psychological behaviour' and 'The sensors do not operate wirelessly, i.e. reliant upon connections being made between the user and a technology'. Literature older than 5 years was excluded from the systematic literature review. Searches were subsequently made in online repositories, such as Google Scholar, IEEE Xplore Digital Library, ACM and international academic institutions. A list of relevant publications was established for each search term, which was then reviewed in detail. 
Detecting Abilities through Smartphone Sensors

Table 1. Mappings of user abilities to smartphone sensors, operating systems and algorithms

\begin{tabular}{|l|l|l|}
\hline \multicolumn{1}{|c|}{ User Abilities } & \multicolumn{1}{|c|}{ Sensors } & \multicolumn{1}{c|}{ OS / Algorithms } \\
\hline Tilting Head & Accelerometer & Android Face Detection (Orientation) \\
getEulerZ()
\end{tabular}

Based on the content of the literature, 
Detecting Abilities through Smartphone Sensors

Table 1 was created to map the user abilities defined in the Framework, to sensor technologies and compatible operating systems (OS) and algorithms. This knowledge was utilised to develop the user interface for the SmartAbility Application.

\section{Ease of Action Threshold Establishment}

Preliminary trials were conducted to establish the Ease of Action boundaries for the blowing, blinking, smiling, speaking and walking abilities. The objectives of the trials were to ascertain numerical values for the boundaries, which could then be defined in the application code. All of these preliminary trials were conducted by the same 10 participants who had typical physical abilities to obtain the boundaries for each ability.

To establish the noise threshold boundaries for sucking in and blowing out air, experiments were performed with ten participants who were performing the tasks with an Android device. The device was running a sound meter application to provide a decibel reading for the movement of air across the microphone. For sucking in, the results were between $52 \mathrm{~dB}$ to $76 \mathrm{~dB}$ and therefore a minimum threshold was selected as $50 \mathrm{~dB}$ for the detection of this physical ability. Sucking out provided a range of results between $46 \mathrm{~dB}$ and $72 \mathrm{~dB}$, resulting in a threshold being selected as $45 \mathrm{~dB}$.

It was decided to detect smiling ability, as our previously conducted EEG trials detected noticeable fluctuations in electronic brain activity when the participant smiled. Even though smiling is not currently a typical method of interaction with assistive technologies, it may become prevalent in the future. The boundaries for detecting blinking and smiling were established using the forward facing camera on a smartphone executing a face detection application. Ten participants conducted each task and the minimum probability for detecting the action was evaluated. For blinking, the probabilities varied 
between 0.16 and 0.32 and it was therefore decided to set the minimum probability for blinking to be 0.30 . A similar trial was conducted for smiling and the probabilities obtained varied between 0.65 and 0.96 . Therefore, a minimum threshold probability was set to 0.90 to guarantee the SmartAbility Application consistently detected the users' ability to smile.

In order to detect the user's ability to speak, it is necessary for the voice recognition software on the smartphone to recognise a voice that has a multitude of variables affecting the sound, in terms of frequency, pitch and volume. Therefore, the phrase used will be complex enough to accurately ascertain whether assistive technology is able to recognise the user's voice. It is important that the phrase naturally reflects those used in the real world, such as "What is your name".

The user's ability to walk can be determined by the step sensor of the smartphone. Preliminary trials were conducted to ascertain the timeframe of the walking task so that an accurate assessment could be made. Ten participants conducted the trial, where each walked for 20, 30, 40, 50 and 60 seconds and a number of steps detected during this time were recorded. It was seen that a timeframe of 40 seconds was sufficient to create boundaries for 'Easy', 'Difficult' and 'Impossible', due to 40 seconds being enough time for a user to walk around a room. A second trial was subsequently conducted where the 10 participants each walked for 40 seconds and the number of steps counted. As it was detected that the participants walked an average of 40 steps, the 'Easy' boundary was defined as 30 steps or above, 'Difficult' boundary defined as between 10 and 30 steps and 'Impossible' boundary defined as less than 5 steps.

\section{SmartAbility Database}

The Application is supported by MySQL database containing the assistive technologies to 
Detecting Abilities through Smartphone Sensors

be recommended by SmartAbility. As these technologies will need updating based on the current availability, it is important that they are stored in a database and not in the Application itself. A database was designed as illustrated in the entity relationship diagram shown in Figure 3. As the Interaction Mediums and Technologies Elements are closely linked, it was decided to combine these elements into a single Assistive Technologies schema in the database. The database therefore contains three schemas, each with a relationship to the 'assistive_technologies' schema, thus mapping Assistive Technologies to Abilities and Manufacturers. This provides the required information for recommendations to be made, based on the users' abilities. It is not necessary to consider physical conditions to provide the assistive technology recommendations, as the characteristics of the users can be defined by their abilities, detected through the smart device sensors. 


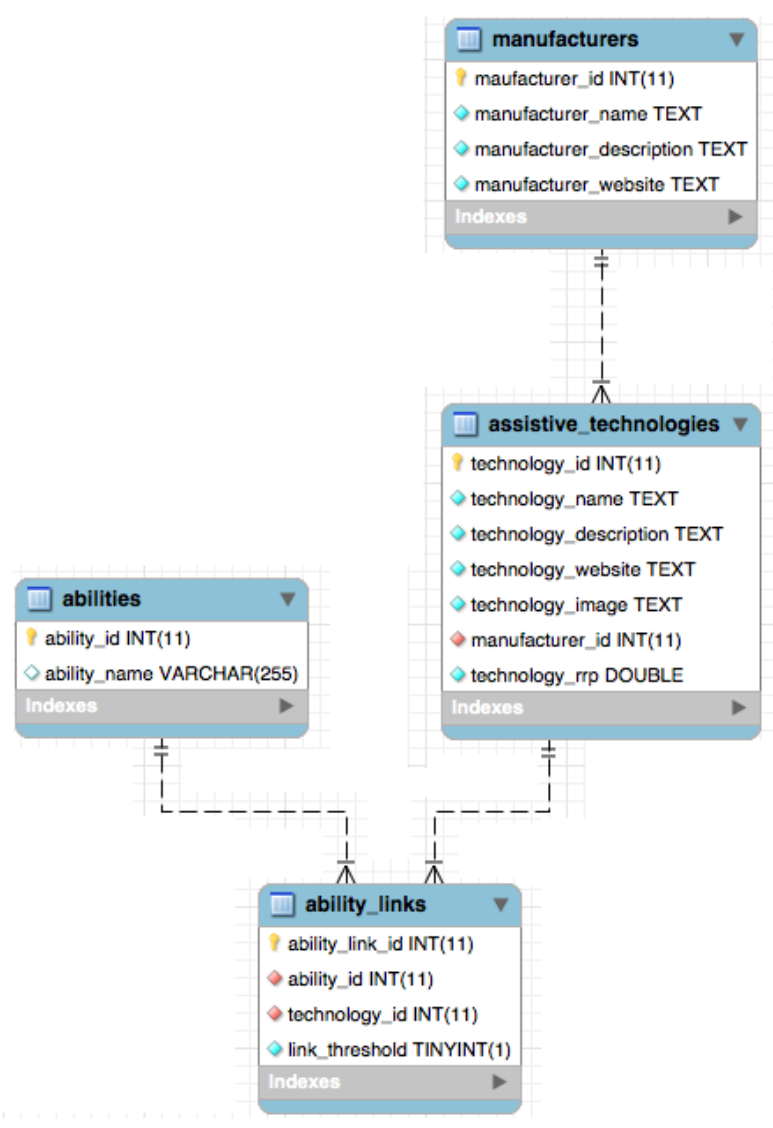

Figure 3. The SmartAbility Entity Relationship Diagram mapping Assistive Technologies to Abilities and Manufacturers

\section{User Interface Design}

The SmartAbility Application consists of the user ability detection interfaces, as well as an administration section that can only be accessed by users who have administrative privileges. These are set when a new SmartAbility user account is created and can only be granted by the supplier of the Application. The welcome screen enables the user to login or register with the Application to commence the evaluation of their user abilities.

The login system adopts the Google Firebase mobile development platform (Google 2020) that allows users to login to the Application using their existing credentials for Facebook, Google or Microsoft. The benefit of Firebase is that users would not need to 
Detecting Abilities through Smartphone Sensors

create a separate account for the Application. However, there is an option to create a standalone SmartAbility account using an email address.

In the administrative section, new assistive technologies can be added to the database and user accounts managed. When adding new assistive technologies, the name, description and external website hyperlink is provided, along with the required user abilities mapped through a series of checkboxes.

Prior to beginning a new ability assessment, the Application requests permission to use the smartphone built-in sensors. The first physical ability captured, utilises the step sensor to count the number of steps taken by the user in 40 seconds, as shown in Figure 4. When the user presses 'Start', a 40 second timer is initialised and the number of steps counted until the timer expires and the device provides a vibration notification.
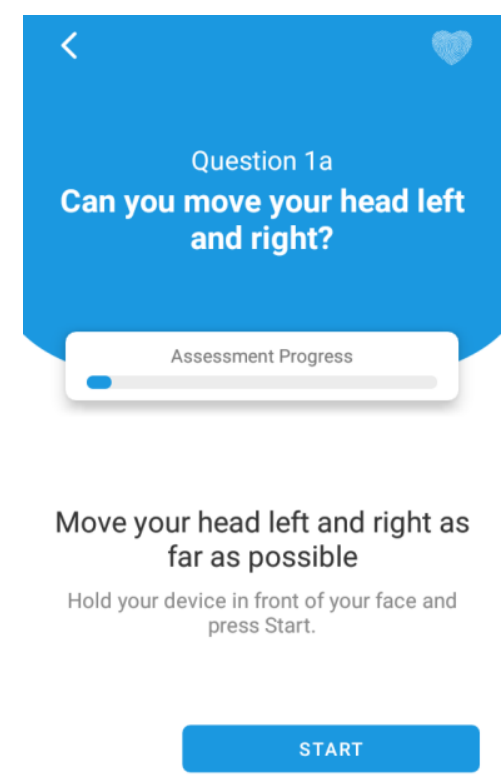

Figure 4. Capturing the user's ability to move their head left and right using the smartphone camera and the faceDetector.Face API 
The subsequent interfaces determine whether the user is able to smile and blink for 5 seconds through utilisation of the RGB camera and faceDetector.Face API. The user's ability to speak is then captured through the Android Voice-to-Text translator, where the user is instructed to repeat the phrase 'What is your name'. The next physical ability to be detected utilised the Android significant motion sensors to determine whether the user can move their left and right shoulders, elbows, wrists and ankles, also shown in Figure 4. The smartphone is able to detect these movements when it is placed on top of the relevant limb. For shoulder, elbow, wrist and ankle movements, the Application provides an animated illustration of how the user should hold the device when performing each task, to ensure the accelerometer and gyroscope can detect the movements. When performing the ankle task, the Application advises the user to attach the device to the foot using an elastic band, although a strap would be developed in the future to accompany the Application.

The final two evaluations determine the ability for the user to move their head and fingers. This was implemented by using the getEulerY() and getEulerZ() commands, relating to head movements and a MotionEvent class regarding touch gestures, such as down, long press and fling.

The user's abilities to suck in and blow out are then evaluated by instructing the user to perform the action into the microphone of the device, where the output is detected by the noise threshold sensor. The threshold decibel level is set to $50 \mathrm{~dB}$ and $45 \mathrm{~dB}$ for sucking in and blowing out respectively, as determined in the previously conducted trials, described in Section II(B).

The final interface of the Application relates to the abilities that cannot be currently captured by built-in sensors, including gazing, moving the tongue and the user's visual 
Detecting Abilities through Smartphone Sensors

acuity (i.e. seeing). The user selects whether these abilities are 'Easy', 'Difficult' or 'Impossible' to perform, through checkboxes, as in the prototype application.

The data collected from the sensors and checkboxes is processed using the mappings between abilities and technologies defined in Java. Suitable assistive technologies are then recommended to the user.

\section{SmartAbility Application Evaluation}

To assess the usability of the SmartAbility Application and identify potential improvements, an evaluation was conducted at a special educational needs school for pupils with reduced physical abilities.

\section{Participants and Procedure}

The evaluation was conducted by 18 pupils, who were a mixture of genders between the ages of 15 and 19, with four being colour blind. The participants all had a range of physical conditions with sufficient cognitive abilities to understand the evaluation task and Table 2 summarises the demographics. The participants thereby became a representative sample to accurately assess the usability of the SmartAbility Application.

The participants were provided with an information sheet explaining the purpose of the evaluation and the tasks that would be involved. Each evaluation had a maximum duration of 30 minutes, including the completion of SUS and NASA TLX questionnaires. The SUS questionnaire was a paper-based version, whereas the NASA TLX questionnaire was completed using a freely available Application developed by NASA (2020). This Application contains 20 point rating scales for each workload type that the user taps to indicate their experienced workload. The questionnaire concluded with participants 
Detecting Abilities through Smartphone Sensors

providing qualitative feedback and any suggestions for improvement. 
Detecting Abilities through Smartphone Sensors

Table 2. User Demographics of the Participants of the SmartAbility Application Evaluation

\begin{tabular}{|l|l|}
\hline & \multicolumn{1}{|c|}{ Statistics } \\
\hline Gender & Male -10, Female -8 \\
\hline Average Age & 17 \\
\hline Physical & Attachment Disorder \\
& Autism \\
& Cerebral Palsy \\
& Dandy Walker Syndrome \\
& Duchenne Muscular \\
& Dystrophy \\
& Dyslexia \\
& Epilepsy \\
& Foetal Alcohol Syndrome \\
& Noonan Syndrome \\
\hline Colour Blindness & 4 \\
\hline
\end{tabular}

\section{SUS and NASA TLX}

The usability of the SmartAbility Application was measured by the participants through SUS and NASA TLX questionnaires, completed after the evaluation. The SUS questions were simplified, as the participants would not have sufficient cognitive abilities to understand standard questions. The SUS questionnaire contained 10 statements, each rated on a 5-point Likert scale from 'Strongly Agree' to 'Strongly Disagree'.

The rationale behind selecting SUS as a measurement of usability was to enable participants to provide single scores for each question, thus calculating SUS scores between 0 and 100 (Bangor et al. 2008). The SUS scores can be analysed using the Adjective Rating Scale (Bangor et al. 2009). This scale provides a description of the usability of the system, ranging from 'Worst Imaginable Usability' (a mean score of less than 12.5) to 'Best Imaginable Usability' (a mean score of greater than 90.9). The NASA TLX questionnaire assessed the workload experienced during the interaction with the SmartAbility Application, in terms of Physical, Mental, Temporal, Performance, Effort and Frustration 
Detecting Abilities through Smartphone Sensors

demands. NASA TLX is a well-established method to analyse workload, which requires minimal amount of training (Stanton et al. 2003).

The Subjective Workload Dominance Technique (SWORD) was considered as an alternative method to rate the workload dominance of one task against another (Stanton et al. 2003). However, this was deemed to be unsuitable, as it only provided a rating for tasks that created a greater workload compared to others and did not calculate an overall rating of the participant's workload. Therefore, this could not be used to evaluate the SmartAbility Application, as the usability of the entire Application needed to be assessed, rather than the workload differences between the performed tasks (Salmon et al. 2003).

\section{Results}

\section{SUS}

Through analysis of the SUS questionnaire, $89 \%$ of participants stated that the Application was easy to use and they were not required to learn a great number of skills before using the Application. Therefore, the support of another individual to explain the Application was not necessary. However, some participants found the buttons difficult to press and certain abilities challenging. Overall, all participants using the Application were very satisfied.

The SUS scores were interpreted by using the Adjective Rating Scale and the SmartAbility Application achieved a score of 72.5 ('Good Usability'). This identified that the users found the Application easy to use, although there were aspects they found challenging. 
Detecting Abilities through Smartphone Sensors

\section{NASA TLX}

Figure 5 contains a box plot comparison of the NASA TLX workloads experienced when participants interacted with the SmartAbility Application.

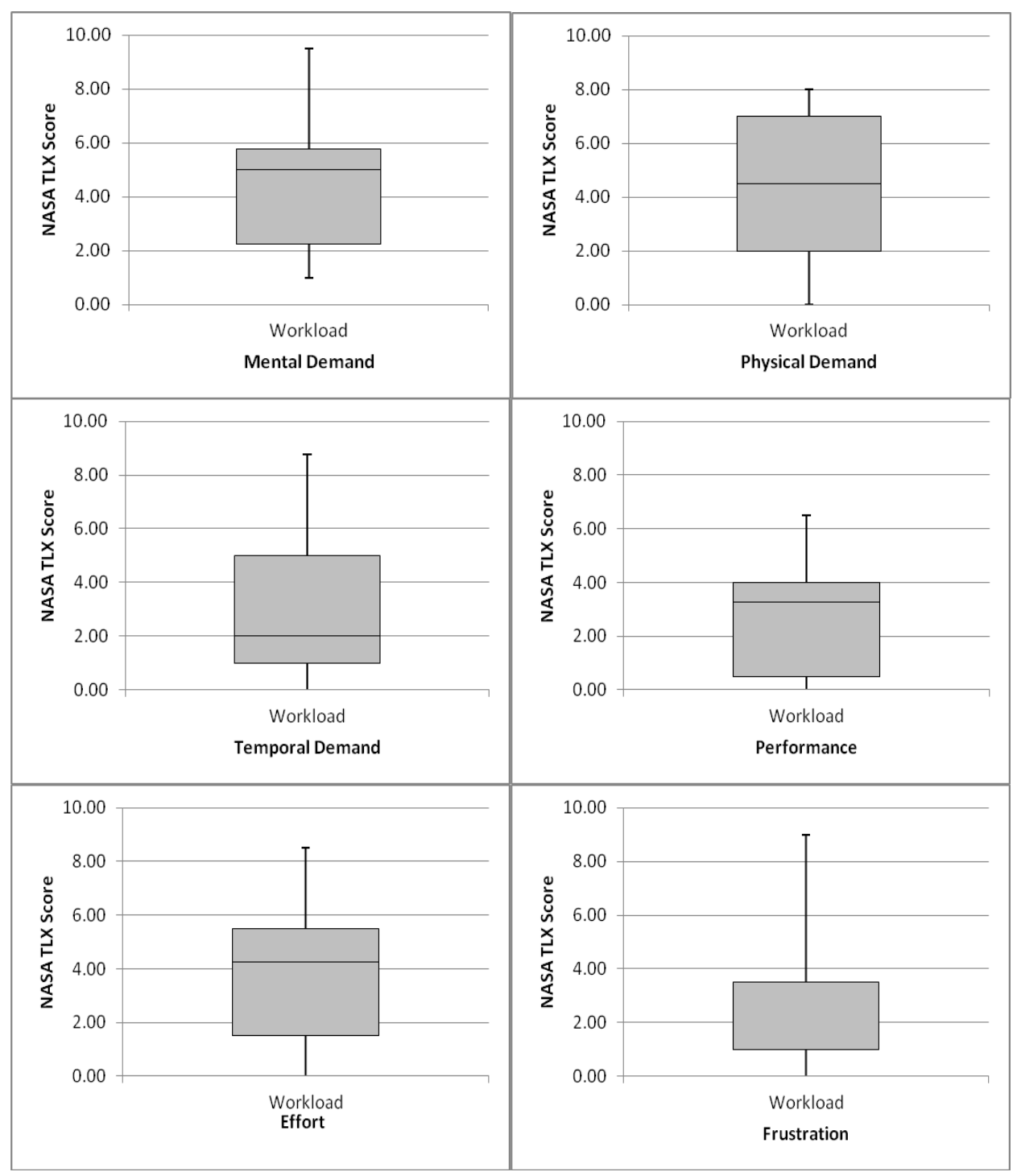

Figure 5. Comparing Physical Demand, Mental Demand, Temporal Demand, Performance Effort and Frustration workloads when interacting with the SmartAbility Application 
Detecting Abilities through Smartphone Sensors

The medians illustrated by the central line on the box plots illustrate that the SmartAbility Application achieved reasonably low NASA TLX scores in all workload categories during participant interaction. There was an average mental demand score of 5, as some of the participants found the task instructions challenging to comprehend, due to their mental abilities. There was a greater range of workload scores for physical demand, as some participants found the tasks easier to perform than others, depending on their physical abilities. The participants did not feel anxious when using the Application, which was reflected in the low temporal demand scores. Although there were specific durations for some tasks, there was not an overall time limit for the ability assessment and therefore participants did not feel under pressure. As all participants could successfully interact with the Application and obtain recommendations of assistive technologies, there was a 'Good Performance' score. Effort scores were higher, as some participants had to work harder to use the Application, as their reduced abilities resulted in the tasks being challenging. The facility to repeat tasks reduced the frustration levels experienced, as participants can have multiple attempts to complete them.

The results from the evaluation of the SmartAbility Application identified areas of improvement to be considered as future work.

\section{Discussion}

The aim of the SmartAbility Framework is to recommend assistive technologies based on the physical abilities of the user, in terms of the actions that can be performed independently. It is therefore conforms to the ability-based design principles and the knowledge was obtained through literature reviews, previously conducted feasibility trials and controlled usability evaluations. A notable evaluation involved head tracking, where it 


\section{Detecting Abilities through Smartphone Sensors}

was highlighted that the suitability of assistive technology was dependent on the ROM of the user, rather than their physical conditions.

To disseminate the Framework to the assistive technology domain, a smartphone application was developed that utilised built-in sensors of smart devices to detect user abilities. Following a systematic literature review into built-in sensors, it was decided that the Android platform offered greater capabilities to measure abilities through existing algorithms. Prior to development of the Application, it was necessary to combine the Interaction Mediums and Technologies element into a single Assistive Technologies element, which is the central schema for the database. These elements were combined as they were closely linked, whereby assistive technologies can be controlled by a variety of interaction mediums.

The Application is able to detect all user abilities included in the Framework, with the exception of eye gazing, seeing, biting and moving the tongue, as built-in sensors do not currently exist that can measure these abilities. The Application consisted of a separate user interface for each ability and included instructions on the method of interaction with the Application. This represents a novel approach whereby the physical abilities of users can be detected with minimal levels of manual input, by using Android sensors. A controlled usability evaluation was conducted with 18 pupils at a special educational needs school, who had varying conditions resulting in reduced physical abilities. SUS and NASA TLX questionnaires were completed, highlighting that the Application achieved the score of 72.5 on the Adjective Rating Scale, indicating 'Good Usability'. There was positive feedback from all 18 participants who enjoyed using the Application, in particular the hand, head and foot tasks. Comments from the participants included that the Application "makes it easy to discover new technologies" and "could be suitable for a wide range of 
disabilities". It was also stated in the feedback that the Application was self-explanatory and quick to use, with sufficient time allowed for each task.

Analysis of the NASA TLX results identified that the Application was quite physically demanding for the users. Through the participants' feedback, the factors that adversely affected the usability were highlighted. Participants highlighted that the increased levels of physical demands were caused by small buttons that were challenging for people with reduced finger dexterity and visual impairments. Also, certain tasks were difficult for some users to perform, such as blowing out and limb movements where the participants had limited ROM. There were also some practical issues raised with the Application, such as a need for a larger smartphone screen and a strap on the phone to assist when performing the tasks.

Based on the feedback from the evaluation, the button and text sizes were increased on the interfaces to improve usability. 'Skip' buttons were introduced on all ability interfaces, so that if the user is not able to perform a specific ability, they can proceed to the next task. It was observed during the evaluation that some tasks had excessive durations. The walking task duration was reduced from 40 seconds to 20 seconds and the durations of the smiling, sucking and blowing out tasks were decreased from 10 seconds to 5 seconds. Additional trials were conducted and it was found that all 10 participants could successfully complete the tasks within the shorter durations. When these durations were integrated into the Application, the efficiency of the assessment was improved, as users were not waiting for the durations to elapse.

The SmartAbility Application enables people with reduced physical ability to discover assistive technologies that could improve their quality of life. The Application could be used by HCPs and charity representatives during the rehabilitation of their clients 
with reduced physical abilities. The abilities of the clients would be inputted into the Application, in order to receive recommendations of suitable assistive technologies. This would replace the existing manual assessment methods adopted by some charities. The benefit of this approach is that users receive recommendations that are suitable to their physical abilities. This reduces the likelihood of the users subsequently purchasing products that are not suitable and unlikely to be used.

\section{Summary}

Designing and developing the SmartAbility Application has resulted in lessons learnt that can be applied to future application developments involving built-in sensors and people with reduced abilities. In order to ensure that the sensors captured the required abilities, the thresholds needed to be considered in terms of the levels indicating Easy, Difficult and Impossible for each ability. We found that the step sensor did not always capture all steps taken and is dependent on the position that the smartphone was held in relation to the user's body. Greater reliability is achieved when the device was how close to the person's chest, rather than away from the body. In order for the microphone to be a suitable sensor to detect a user's speaking ability, it is important to consider the phrase to be spoken. Performance is increased with shorter phrases, however, a sentence should be used to provide an accurate representation of general speech. Left and right head movements are more reliably detected than vertical movements but blinking and smiling could consistently be detected. It was realised that the capability of the smartphone to detect limb movements is dependent on ensuring that the device was securely attached to the relevant limb. This creates a challenge; even for able bodied participants, as there is a risk of dropping the device and we recommend that the device should be attached using a strap. The 
combination of adopting NASA TLX, SUS and the Adjective Rating Scale, enables a usability assessment to be conducted, assessing a variety of workloads and producing an overall score of the usability of the Application. This emphasises the importance of involving the intended user community, i.e. people with reduced physical abilities, to obtain an accurate assessment. When conducting evaluations with people who have disabilities, it may require a greater amount of time to be allocated compared with able bodied participants and it is essential to ensure that the language used in surveys or information documents is clear to understand.

\section{Future Work and Conclusions}

The SmartAbility Application will be enhanced in subsequent iterations of the development. Audible questions will be included to assist users who have difficulty in reading text. As some users operate smart devices through third party input devices, testing will be conducted on the Application, to ensure that efficient interface navigation can be achieved with these devices.

Additional assistive technologies will be included in the Application that will be mapped to the required abilities and included in the database. To establish the mappings, experimentations would need to be performed with the products that involve the user community of people with reduced physical ability. It is anticipated that the Application will develop into a product promotion tool for assistive technology manufacturers and charities, whereby their products can be promoted to the user community through customised applications. Each application would include the manufacturers or charities logos, product images and descriptions. The currently developed Application, as well as future manufacturer or charity specific applications, would initially be made freely 
available to the user community. This would be via digital distribution platforms or through websites of associations connected with reduced physical abilities. Depending on the popularity of the SmartAbility Application, a download fee could be introduced.

It would be necessary to conduct a further validation phase of the Application to evaluate the usability and determine the usefulness of the assistive technology recommendations to people with reduced physical abilities. This validation phase would involve representatives from assistive technology manufacturers or charities and their clients with reduced physical abilities. The manufacturers or charities would be provided with the SmartAbility Application through the Google Play Store, so that they can disseminate to their employees and clients. The participants of the validation would be invited to complete an online survey to provide feedback, suggestions of improvements or additional functionality, which will be considered in future developments of the SmartAbility Application. The usefulness of the recommendations could be measured in terms of abandonment rates of the assistive technologies after specific timeframes, such as a week, month and year (Leckie 2010). As an additional method of validation, the recommendations provided from the Application can be compared to recommendations provided by a human expert at charities who provide assistive technology recommendations. The results of these comparisons will ascertain the efficiency of SmartAbility.

The aim of SmartAbility is to supplement the assistive technology assessments currently performed by domain experts at manufacturers or charities. The limitation of an assessment conducted by an Application is that an individual with reduced physical ability may prefer the human interaction of an assessment, or may not be able to use an Android device. However, the Application could be used as an initial assessment tool, prior to 
manufacturer or charity representatives visiting the individual, or vice versa. The benefits are a reduction on travelling time and costs, potentially enabling a greater number of assessments to be performed. The individual could subsequently arrange a manual assessment with the manufacturer or charity, to obtain further information or to have a demonstration of the recommended assistive technologies.

An education technology version of the Application will be developed that recommends assistive technologies to be used in the education sector. This Application, named 'AT4SEND', will be suitable for people who have Special Educational Needs and Disabilities, i.e. both reduced physical and cognitive abilities. The existing physical abilities in the SmartAbility Framework will be supplemented with cognitive abilities stated in the ICF Checklist (World Health Organization 2003). The recommendations provided by this Application will improve pupils' access to education through the use of assistive technology. This will support the UK Government's strategy, 'Realising the potential of technology in education' (Department for Education 2019). Once AT4SEND is developed, we plan to conduct usability evaluations involving people with reduced physical and cognitive abilities and Additional Learning Support teams at special educational needs schools, mainstream schools and higher educational institutions including Universities.

The SmartAbility Framework enables assistive technologies to be recommended as part of the rehabilitation process of people with reduced physical abilities. The recommendations vary depending on individuals' user abilities, as there is not a 'single solution to fit multiple needs', comparable to the 'One Size Fits All' Information Technology concept (Adams 2017). The knowledge behind the Framework was established through literature reviews of user abilities, interaction mediums and technologies, and the Framework was designed to ensure conformance to the principles of ability-based design. 
Detecting Abilities through Smartphone Sensors

The SmartAbility Application was developed through the novel use of the Android sensors, to minimise the manual input required to detect physical abilities. The Application provides a route to dissemination of SmartAbility and through the continual development of the Framework, assistive technology awareness will be promoted during the rehabilitation of the user community. This will enhance and improve the quality of lives for people with reduced physical abilities.

\section{Word Count: 7,532}

Acknowledgments: We thank the participants of the controlled usability evaluation of the SmartAbility Application, who provided informative feedback and suggestions for improvements. The authors report no conflict of interest.

\section{REFERENCES:}

Adams, J., 2017. The High Cost of a One Size Fits All Technology Approach [online]. Available from: https://blogs.poly.com/high-cost-one-size-fits-technology-approach [Accessed 10 October 2020].

Akao, Y., 1990. QFD: Quality Function Deployment - Integrating Customer Requirements into Product Design. New York: Productivity Press. Android Developers, 2020. Detecting Common Gesture [online]. Available from: https://developer.android.com/training/gestures/detector.html [Accessed 10 October 2020].

Android Developers, 2020. Motion Sensors [online]. Available from: https://developer.android.com/guide/topics/sensors/sensors_motion.html [Accessed 10 October 2020].

Assistive Technology Industry Association, 2020. What is AT? [online]. Available from: https://www.atia.org/at-resources/what-is-at/ [Accessed 10 October 2020]. 
Detecting Abilities through Smartphone Sensors

Bangor, A., Kortrum, P. And Miller, J., 2008. An Empirical Evaluation of the System Usability Scale. Human-Computer Interaction, 24 (6), 574-594.

Bangor, A., Kortrum, P. And Miller, J., 2009. Determining What Individual SUS Scores Mean: Adding an Adjective Rating Scale. Usability Studies, 4 (3), 114-123.

Biswas, P. and Robinson, P., 2008. Automatic evaluation of assistive interfaces. In:

Proceedings of the $13^{\text {th }}$ International Conference on Intelligent User Interfaces. Available from: https://doi.org/10.1145/1378773.1378806

Brocket, C.L. and Chapman, G.J., 2016. Biomechanics of the ankle. Orthopaedic Trauma, 30 (3) 232-238. Available from: https://dx.doi.org/10.1016\%2Fj.mporth.2016.04.015

Brooke, J., 1996. SUS: A quick and dirty usability scale. In: P.W. Jordan, B. Thomas, I.L. McClelland, B. Weerdmeester, eds. Usability Evaluation in Industry. London: CRC Press, $189-194$

Department For Education, 2019. Realising the potential of technology in education: A strategy for education providers and the technology industry [online]. Available from: https://assets.publishing.service.gov.uk/government/uploads/system/uploads/attachment_da ta/file/791931/DfE-Education_Technology_Strategy.pdf [Accessed 10 October 2020].

Francis, W. J. 2013. Make your next Android app a good listener [online]. Available from: http://www.techrepublic.com/blog/software-engineer/make-your-next-Android-app-a-goodlistener/ [Accessed 10 October 2020].

Gallagher, B. and Petrie, H. 2013. Initial Results from a Critical Review of Research on technology for Older and Disabled People. In: Proceedings of The $15^{\text {th }}$ ACM SIGACCESS International Conference on Computers and Accessibility, 21-23 October 2013 Bellevue. Available from: https://doi.org/10.1145/2513383.2513417 
Detecting Abilities through Smartphone Sensors

Gates, D. H., Walters, L. S., Cowley, J., Wilken, J. M. and Resnik, L. 2015. Range of Motion Requirements for Upper-Limb Activities of Daily Living. American Journal of Occupational Therapy, 70 (1).

Google, 2020. Firebase helps mobile and web app teams succeed [online]. Available from: https://firebase.google.com/ [Accessed 10 October 2020].

Google Developers, 2020. Face Detection Concepts Overview [online]. Available from: https://developers.google.com/vision/face-detection-concepts [Accessed 10 October 2020].

Keilhofner, G., 2006. Research in Occupational Therapy: Methods of Inquiry for Enhancing Practice. Philadelphia: F.A. Davis Company.

Kelley, D. And Harfield, B., 2006. The designer's stance. In: J. Bennett, L. De Young, B. Hartfield, eds. Bringing Design to Software. Boston, MA: Addison-Wesley, 151-171.

Khadilkar, L. MacDermid, J.C., Sinden, K.E., Jenkyn, T.R., Birmingham, T.B. and Athwal, G.S., 2014. An analysis of functional shoulder movements during task performance using Dartfish movement analysis software. Shoulder Surgery, 8 (1), 1-9. Available from: https://dx.doi.org/10.4103\%2F0973-6042.131847

Kostanjsek, N., 2011. Use of The International Classification of Functioning, Disability and Health (ICF) as a conceptual Framework and common language for disability statistics and health information systems, Public Health, 11 (4) 1-6. Available from: https://doi.org/10.1186/1471-2458-11-S4-S3

Leckie, C., 2010. The abandonment of technology [online]. Available from: http://www.resilience.org/stories/2010-10-16/abandonment-technology/ [Accessed 10 October 2020]. 
Detecting Abilities through Smartphone Sensors

National Aeronautics and Space Administration, 2020. NASA TLX: Task Load Index

[online]. Available from: https://humansystems.arc.nasa.gov/groups/tlx/

Origin Instruments Corporation, 2020. Sip and Puff Switch Solutions [online]. Available from: http://www.orin.com/access/sip_puff/ [Accessed 10 October 2020].

Salmon, P., Stanton, N.A., Walker, G.H., 2003. Human Factors Design Methods Review [online]. Available from:

https://eprints.soton.ac.uk/368316/1/_soton.ac.uk_ude_personalfiles_users_jr1d11_mydes ktop_ePrints_hf-design-methods-review.pdf [Accessed 10 October 2020].

Siddaway, A., 2020. What is a systematic literature review and how do I do one? [online]. Available from:

https://pdfs.semanticscholar.org/2214/2c9cb17b4baab118767e497c93806d741461.pdf [Accessed 10 October 2020].

Sloan, D., Atkinson, M. T., Machin, C. and Li, Y. 2010. The potential of adaptive interfaces as an accessibility aid for older web users. In: Proceedings of the International Cross-Disciplinary Conference on Web Accessibility, 26-27 April 2010 Raleigh. Available from: https://doi.org/10.1145/1805986.1806033

Stanton, N. A. Salmon, P. M., Rafferty, L. A., Walker, G. H., Baber, C. and Jenkins, D. P., 2003. Human Factors Methods: A Practical Guide for Engineering and Design. London: CRC Press.

StatCounter, 2020. Mobile Operating System Market Share Worldwide [online]. Available from: http://gs.statcounter.com/os-market-share/mobile/worldwide [Accessed 10 October 2020].

The World Bank, 2020. Disability Inclusion [online]. Available from: http://www.worldbank.org/en/topic/disability [Accessed 10 October 2020]. 
Detecting Abilities through Smartphone Sensors

Usability.gov, 2020. Focus Groups [online]. Available from:

https://www.usability.gov/how-to-and-tools/methods/focus-groups.html [Accessed 10

October 2020].

Virtual Reality Society, 2017. Understanding Sensors: Magnetometers, Accelerometers and Gyroscopes [online]. Available from: https://www.vrs.org.uk/virtual-reality-gear/motiontracking/sensors.html [Accessed 10 October 2020].

Whittington, P. and Dogan, H. 2016. SmartDisability: A smart system of systems approach to disability. In: Proceedings of the 11th System of Systems Engineering Conference, 12-16 June 2016 Kongsberg. New York: IEEE Press. Available from: https://doi.org/10.1109/SYSOSE.2016.7542943

Whittington, P. and Dogan, H. 2016. A SmartDisability Framework: enhancing user interaction. In: Proceedings of the 30th International BCS Human Computer Interaction Conference, 11-15 July 2016 Bournemouth. London: British Computer Society. Available from: https://dx.doi.org/10.14236/ewic/HCI2016.24

Whittington, P. and Dogan, H., 2016. SmartPowerchair: Characterisation and Usability of a Pervasive System of Systems. Human Machine Systems, 47 (4), 500-510. Available from: https://doi.org/10.1109/THMS.2016.2616288

Whittington, P., Dogan, H. and Phalp, K, 2015. Evaluating the Usability of an Automated Transport and Retrieval System. In: Proceedings of the 2015 International Conference on Pervasive and Embedded Computing and Communication Systems, 11-13 February 2015 Angers. Available from: https://ieeexplore.ieee.org/document/7483733/

Whittington, P., Dogan, H. and Phalp, K, 2015. SmartPowerchair: to boldy go where a powerchair has not gone before. In: Proceedings of Ergonomics and Human Factors, 13-16 April 2015 Daventry. Available from: 
Detecting Abilities through Smartphone Sensors

https://books.google.co.uk/books?id=zT9ZDwAAQBAJ\&lpg=PA233\&ots=upyCMBxp6b $\& \mathrm{dq}=$ SmartPowerchair\%3A\%20to\%20boldy\%20go\%20where\%20a\%20powerchair\%20ha s\%20not\%20gone\%20before\%E2\%80\%9D\&lr\&pg=PA233\#v=onepage\&q\&f=false

Whittington, P., Dogan, H., Jiang, N. and Phalp, K. 2018. Automatic Detection of User Abilities through the SmartAbility Framework. In: Proceedings of the 32nd International BCS Human Computer Interaction Conference, 2-6 July 2018 Belfast. Available from: http://eprints.bournemouth.ac.uk/30955/1/Automatic\%20Detection\%20of\%20User\%20Abi lities\%20through\%20the\%20SmartAbility\%20Framework\%20-\%20BHCI2018_paper_133.pdf

Wobbrock, J.O. Gajos, K.Z., Kane, S.K. and Vanderheiden, G.C., 2018. Ability-based design. Communications of the ACM, 61 (6), 62-71. Available from: https://doi.org/10.1145/3148051

World Health Organization, 2001. International Classification of Functioning, Disability and Health (ICF) Framework [online]. Available from: http://www.who.int/classifications/icf/en/ [Accessed 10 October 2020].

World Health Organization, 2003. ICF Checklist for International Classification of Functioning, Disability and Health [online]. Available from: https://www.who.int/classifications/icf/icfchecklist.pdf?ua=1 [Accessed 10 October 2020]. 